\title{
Breast Cancer Survival According to Number of Nodes Removed
}

\author{
David N. Krag, MD, FACS, and Richard M. Single, PhD
}

\begin{abstract}
Background: Results from randomized trials indicate a 5.4\% survival advantage associated with axillary dissection. To gain insight on survival outcomes when less than an axillary dissection is performed, we performed a retrospective analysis to determine survival outcome for node-negative and node-positive breast cancer patients when a variable number of nodes were excised.

Methods: The data analyzed in this paper are from the Surveillance, Epidemiology, and End Results (SEER) database, from which 72,102 patients were selected whose breast cancer had been diagnosed in 1988 or later and who were aged 40-79 years at diagnosis, had a single primary lesion, and had 0 to 3 positive lymph nodes. Cases were separated into age groups (40 to 49 and 50 to 79 years), and node-negative cases were separated from those with one to three positive nodes.

Results: This analysis indicates that even when all regional lymph nodes are pathologically negative, the number of nodes removed is associated with survival. In the group of breast cancer patients who had one to three pathologically positive nodes, as with the node-negative group, the higher the number of nodes removed, the greater the survival. The hazard rate for death in the node-negative group was roughly $5 \%$ less for each additional five nodes removed. For the node-positive group, the hazard rate for death was between $8 \%$ and $9 \%$ less for each additional five nodes removed.

Conclusions: This retrospective study supports the notion that removal of regional nodes, even when such nodes are interpreted as pathologically negative, is important for the long-term survival of breast cancer patients.
\end{abstract}

Key Words: Axilla—Breast cancer-Lymph nodes-Survival.

There is a common conception that axillary surgery has no impact on survival of breast cancer patients. In North America this notion is based primarily on the results of the National Surgical Adjuvant Breast and Bowel Project (NSABP) B-04 clinical trial, in which clinically node-negative patients were randomized to undergo axillary dissection (AD) or no axillary resection. ${ }^{1}$

\footnotetext{
Received March 11, 2003; accepted August 11, 2003.

From the College of Medicine (DNK) and Department of Medical Biostatistics (RMS), University of Vermont, Burlington, Vermont.

The authors both contributed to conception and design; acquisition of a substantial portion of data; analysis and interpretation of data; drafting of the manuscript; and critical revision of the manuscript for important intellectual content and statistical expertise. Each author declares no competing interests.

Address correspondence and reprint requests to: David N. Krag, MD, FACS, S. D. Ireland Professor of Surgical Oncology, College of Medicine, University of Vermont, Given Building, Room E309, Burlington, VT 05405; Fax: 802-656-5833; E-mail: david.krag@uvm.edu.

Published by Lippincott Williams \& Wilkins @ 2003 The Society of Surgical Oncology, Inc.
}

In the B-04 trial, among patients who underwent an axillary resection, the observed survival rate was higher (by $4 \%$ ) than among patients who did not undergo an AD. The accrued number of patients was not sufficient to detect a survival difference of this magnitude, so even though there was an observed improvement of $4 \%$, this observation did not reach statistical significance. It appears that given the number of patients enrolled and the number of events, the study would not have been able to show an absolute survival difference below 5\% to $10 \% .^{2,3}$

Five other clinical trials in which patients were randomized to undergo axillary resection or observation also revealed a higher observed survival number in the axillary resection group. The reported increase in survival of the $\mathrm{AD}$ group ranged from $4 \%$ to $16 \% .^{1,4-8}$ Taken together, the overall survival benefit associated with $\mathrm{AD}$ in all six trials was $5.4 \% .^{9}$ 
It would seem to be a simple idea that if we could identify those patients who had cancer in their regional nodes and who would have decreased survival rates, we could limit regional node surgery to that group of patients. However, randomized trials comparing observation to regional node resection have not provided that information. The status of the sentinel node (SN) may provide that information, because the presence or absence of regional node metastases can be noted without performing a complete $\mathrm{AD}$. On the basis of numerous studies in which AD followed SN surgery, it appears that $\mathrm{SN}$ status is an excellent predictor of the overall node status, as determined by pathological evaluation. However, we do not yet have long-term randomized survival data comparing $\mathrm{SN}$ resection only to regional node resection.

Until the existing randomized SN trials are complete, we will not have definitive information regarding survival outcomes following this new procedure. Resecting no lymph nodes is associated with a negative impact on survival and long-term regional control. No randomized clinical trials comparing axillary dissection to partial axillary dissection have had sufficient patient accrual for accurate determination of relatively small potential survival differences. Therefore, the impact on survival of a partial or incomplete $\mathrm{AD}$ is not known. The $\mathrm{SN}$ status is not always accurately determined, and existing analytical methods used by pathologists can be considered an uncontrolled experiment in sampling (and therefore incomplete).

We asked, what would the survival outcome be when only a limited number of lymph nodes were excised? We analyzed a large database and reviewed available literature. The study reported here was designed to analyze survival outcome in relation to the total number of nodes excised when (1) all nodes are negative on pathological examination and (2) a limited number of nodes (1 to 3) are positive on pathological examination. The data from this analysis would potentially help frame questions regarding SN surgery in which a pathologically negative $\mathrm{SN}$ might be considered equivalent to an all-nodes-negative status. The all-nodes-negative analysis relates to the current NSABP B-32 trial, in which survival will be compared between node-negative patients who underwent SN resection only (few nodes excised and all negative) and patients who underwent $\mathrm{SN}$ resection plus $\mathrm{AD}$ (many nodes excised and all negative). This analysis also provides insight into the possible survival outcome among a large number of women who have a limited set of nodes positive but have variable numbers of negative nodes excised. This latter analysis is similar to the major endpoints of the American College of Surgeons Oncol- ogy Group (ACOSOG) trial Z0011, in which patients with pathologically positive SNs are randomized to undergo $\mathrm{SN}$ resection only or $\mathrm{AD}$.

\section{MATERIALS AND METHODS}

\section{Data Source}

The data analyzed in this article are from the Surveillance, Epidemiology, and End Results (SEER) program's public-use CDROM issued in April 2001. The SEER program of the National Cancer Institute collects data on the survival experience of cancer patients with tumor registries in four metropolitan areas and five states. These registries collect data that include tumor location, size, and grade, as well as lymph node status and age of the patient. Treatment data are limited to therapy received in the first 4 months after diagnosis. Since 1988, data have been collected on the number of lymph nodes examined and the number found to be positive.

For this article the term excised or removed is used in place of the term examined, as used in the SEER database, to refer to the number of nodes described in the pathology report. Surgeons typically refer to the number of nodes reported by the pathologist as the number removed rather than the number examined. Our goal is to be consistent with common use, in light of the fact that in any situation the number of nodes reported might not perfectly tally the number of nodes excised.

\section{Case Selection}

For this study we selected patients whose breast cancer had been diagnosed in 1988 or later and who were aged 40-79 years at diagnosis, had a single primary lesion that was microscopically confirmed, and had 0 to 3 positive lymph nodes. In addition, cases involving the following factors were excluded from the study: in situ carcinomas or stage IV disease; tumor size of $>5.0 \mathrm{~cm}$; diagnosis only from an autopsy or death certificate; or survival time not noted. The number of cases meeting the inclusion criteria was 72,102 .

\section{Analysis}

Cases were separated into two age groups (40 to 49 years and 50 to 79 years) according to the patients' age at diagnosis. Node-negative cases were separated from those with one to three positive nodes. Combining these two classifications led to four different groups. Analyses were conducted separately for each of these four groups.

The relationship between the number of nodes removed and the survival experience of the study cases 
was first examined graphically with the Kaplan-Meier method. The number of nodes removed was categorized as 1 to 5,6 to 10,11 to 15 , or 16 or more. The log-rank test was used to determine statistical significance.

In light of the extremely large sample size, significance testing was not the primary goal of the analyses; rather, it was to characterize the relationship between the number of nodes removed and the survival experience in this cohort. Care should be taken if one compares $P$ values from one analysis group to another, because the different groups may have substantially different sample sizes, and small differences that are not medically important may be statistically significant because of the large sample size.

The effect of the number of nodes removed was investigated with Cox models controlling for age, diagnosis year, and tumor size. The number of nodes removed was considered a continuous variable in the Cox models. Tumor size was controlled by stratification because the proportional hazards assumption was not met for this variable.

\section{RESULTS}

Table 1 provides descriptive statistics for the cases that met the selection criteria described above. For each age category considered, the distributions of the number of nodes removed in the node-negative and node-positive groups are not substantially different. However, the average tumor size in the node-positive group is larger than that in the node-negative group in each age category.

Figure 1 shows the Kaplan-Meier curves for the four analysis groups. Larger numbers of nodes removed correspond to a higher survival rate. The worst overall survival experience is seen for those with one to five nodes removed. Overall, a higher percentage of patients survived longer if a greater number of nodes were removed. This pattern is most pronounced in the older node-positive group, but it is evident for each of the groups. Results of the log-rank test comparing the four survival curves were significant $(P<.0001)$ for each of the analysis groups except the node-negative group aged $40-49$ years.

TABLE 1. Descriptive statistics of cases selected

\begin{tabular}{|c|c|c|c|c|c|c|}
\hline No. of nodes positive & Age & Variable & & $\mathrm{n}$ & Mean & SD \\
\hline \multirow[t]{16}{*}{0} & $40-49$ & Tumor size $(\mathrm{cm})$ & & & 1.7 & .9 \\
\hline & & Diagnosis year & & & 1993 & 3.11 \\
\hline & & No. nodes examined & & & 15.4 & 6.8 \\
\hline & & & 1 to 5 & 565 & & \\
\hline & & & 6 to 10 & 2212 & & \\
\hline & & & 11 to 15 & 3804 & & \\
\hline & & & $16+$ & 5316 & & \\
\hline & & & $\overline{\text { Total }}$ & $\overline{11897}$ & & \\
\hline & $50-79$ & Tumor size $(\mathrm{cm})$ & & & 1.65 & .91 \\
\hline & & Diagnosis year & & & 1993 & 3.13 \\
\hline & & No. nodes examined & & & 14.7 & 6.50 \\
\hline & & & 1 to 5 & 2252 & & \\
\hline & & & 6 to 10 & 9436 & & \\
\hline & & & 11 to 15 & 14641 & & \\
\hline & & & $16+$ & 17728 & & \\
\hline & & & Total & 44057 & & \\
\hline \multirow[t]{16}{*}{$1-3$} & $40-49$ & Tumor size (cm) & & & 2.2 & 1.0 \\
\hline & & Diagnosis year & & & 1993 & 3.10 \\
\hline & & No. nodes examined & & & 15.7 & 6.7 \\
\hline & & & 1 to 5 & 170 & & \\
\hline & & & 6 to 10 & 802 & & \\
\hline & & & 11 to 15 & 1480 & & \\
\hline & & & $16+$ & 2083 & & \\
\hline & & & Total & 4535 & & \\
\hline & $50-79$ & Tumor size $(\mathrm{cm})$ & & & 2.1 & 1.0 \\
\hline & & Diagnosis year & & & 1993 & 3.21 \\
\hline & & No. nodes examined & & & 15.0 & 6.6 \\
\hline & & & 1 to 5 & 545 & & \\
\hline & & & 6 to 10 & 2409 & & \\
\hline & & & 11 to 15 & 3849 & & \\
\hline & & & $16+$ & 4810 & & \\
\hline & & & Total & 11613 & & \\
\hline
\end{tabular}


Nodes Positive: 0 , Age: $40-49$

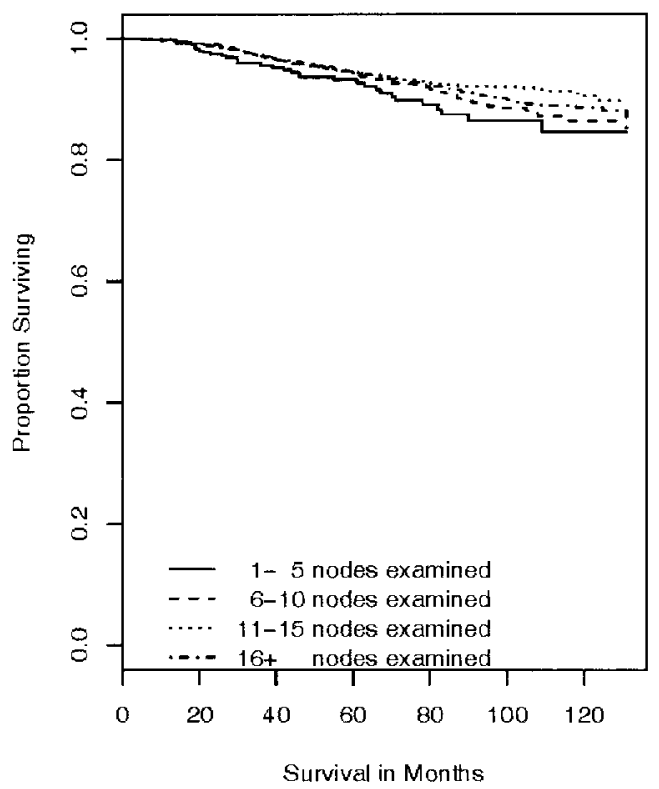

(d)

Nodes Positive: 0, Age: $\mathbf{5 0 - 7 9}$

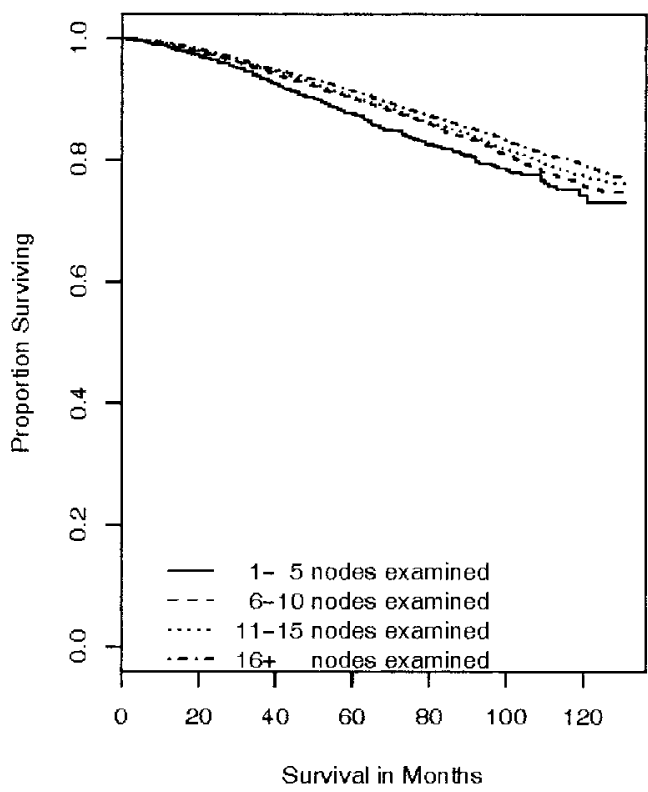

(b)
Nodes Positive: 1-3, Age: 40-49

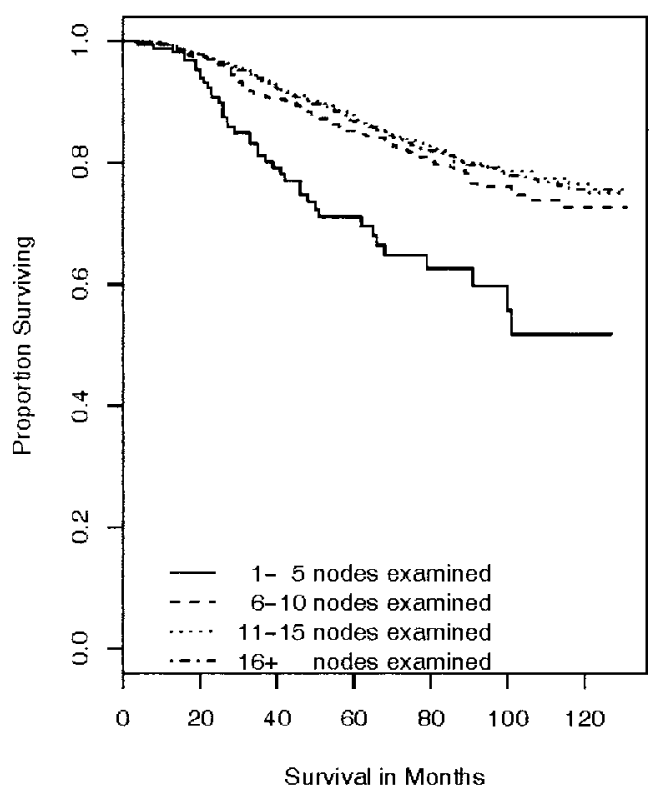

(c)

Nodes Positive: 1-3, Age: 50-79

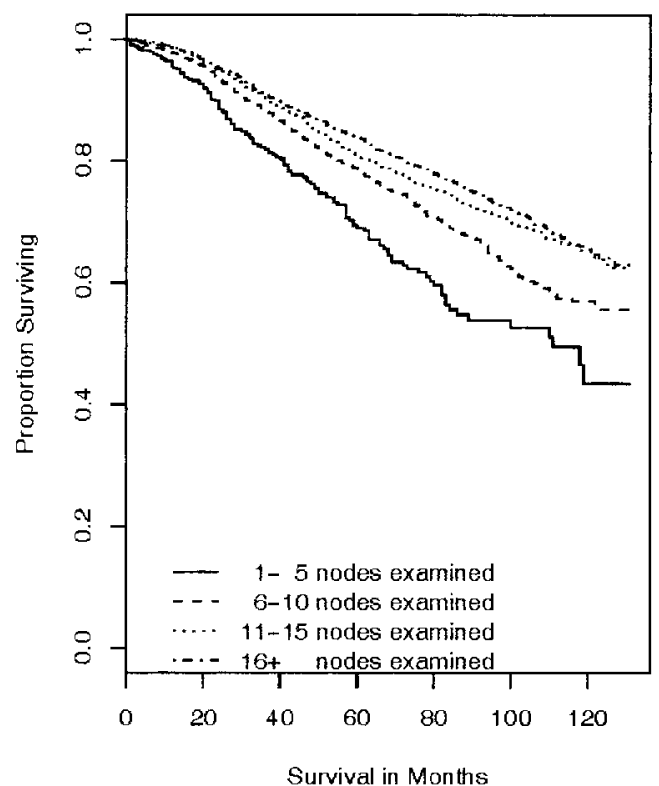

(d)

FIG. 1. Kaplan-Meier curves for the four analysis groups: (a) nodes positive $=0$, age $=40-49$ years, $\log$-rank $P$ value $=.063$; (b) nodes positive $=0$, age $=50-79$ years, log-rank $P$ value $<.0001 ;$ (c) nodes positive $=1-3$, age $=40-49$ years, $\log$-rank $P$ value $<.0001 ;($ d) nodes positive $=1-3$, age $=50-80$ years, log-rank $P$ value $<.0001$.

Table 2 provides hazard ratios for each additional five nodes removed. The hazard ratios, which can be thought of as ratios of hazard rates, are derived from the afore- mentioned Cox models, controlling for the covariates age, diagnosis year, and tumor size. The term hazard rate is used to refer to an incidence rate that varies over time 
TABLE 2. Hazard ratios per five nodes removed

\begin{tabular}{cccc}
\hline $\begin{array}{c}\text { No. of nodes } \\
\text { positive }\end{array}$ & Age & $\begin{array}{c}\text { Hazard } \\
\text { ratio }\end{array}$ & $\begin{array}{c}95 \% \text { Confidence } \\
\text { interval }\end{array}$ \\
\hline 0 & $40-49$ & .955 & $(.901,1.012)$ \\
& $50-79$ & .951 & $(.929, .974)$ \\
$1-3$ & $40-49$ & .920 & $(.672, .979)$ \\
& $50-79$ & .906 & $(.763, .938)$ \\
\hline
\end{tabular}

in survival analysis. The hazard ratio in this setting represents the instantaneous relative risk of death per unit of time for one individual versus another with five fewer nodes removed and with the same values for the set of covariates, given that both individuals have survived to the time of comparison.

The values in Table 2 show that the hazard rate, or instantaneous probability of death per unit of time, is lower for those with more nodes removed. These results indicate that for the node-negative group the hazard rate is roughly $5 \%$ less for each additional five nodes removed. For the node-positive group, the hazard rate is between $8 \%$ and $9 \%$ less for each additional five nodes removed.

Table 3 displays the survival percentage at 10 years for patients who had less than 10 versus at least 10 nodes removed in the four analysis groups. A larger number of nodes removed was associated with a better survival experience for each of the analysis groups. The log-rank $P$ values for comparison of the two categories of nodes removed are similar to those for the categorization used in Figure 1.

\section{DISCUSSION}

This analysis of the SEER database indicates that even when all regional lymph nodes are pathologically negative, the number of nodes removed is significantly asso-

TABLE 3. Survival according to age and a cutoff of 10 nodes excised in node-negative and 1-3 node-positive women with breast cancer

\begin{tabular}{ccccc}
\hline $\begin{array}{c}\text { No. of nodes } \\
\text { positive }\end{array}$ & $\begin{array}{c}\text { Age } \\
\text { group }\end{array}$ & $\begin{array}{c}\text { No. of nodes } \\
\text { removed }\end{array}$ & $\begin{array}{c}10-\mathrm{y} \\
\text { survival }\end{array}$ & $\begin{array}{c}\text { Log-rank } \\
P \text { value }^{a}\end{array}$ \\
\hline 0 & $40-49$ & $<10$ & $86 \%$ & .054 \\
0 & $40-49$ & $\geq 10$ & $89 \%$ & \\
0 & $50-79$ & $<10$ & $75 \%$ & $<.0001$ \\
0 & $50-79$ & $\geq 10$ & $78 \%$ & \\
$1-3$ & $40-49$ & $<10$ & $66 \%$ & $<.0001$ \\
$1-3$ & $40-49$ & $\geq 10$ & $76 \%$ & \\
$1-3$ & $50-79$ & $<10$ & $54 \%$ & $<.0001$ \\
$1-3$ & $50-79$ & $\geq 10$ & $65 \%$ & \\
\hline
\end{tabular}

${ }^{a}$ The log-rank $P$ value reports the significance of the comparison of survival for the $<10$ and $\geq 10$ nodes removed groups in each analysis group. ciated with survival. The higher the number of pathologically negative nodes removed, the greater the survival rate. This observation was statistically significant for the postmenopausal age group. The premenopausal age group trended toward improved survival with higher number of pathologically negative lymph nodes removed, but this result did not reach statistical significance.

In the group of breast cancer patients who had 1 to 3 pathologically positive nodes, the number of nodes was associated with an even greater survival difference than with the node-negative group. As with the node-negative group, the higher the number of nodes removed, the greater the survival rate. This observation was statistically significant for all age groups.

The observed survival difference related to the number of lymph nodes should be put in perspective with the findings associated with other major breast cancer treatment modalities. For example, for women $<50$ years of age, polychemotherapy results in 10-year survival improvement of $7 \%$ to $11 \%$ (for node-negative and nodepositive groups, respectively), and for women $>50$ years of age, a survival improvement of $2 \%$ to $3 \%$ (for nodenegative and node-positive groups, respectively). ${ }^{10}$ In contrast, our data analysis showed that among women who were $<50$ years of age and had 1 to 3 positive nodes, removal of $\geq 10$ nodes compared with $<10$ nodes resulted in 10-year survival improvement of $10 \%$. For women who were $\geq 50$ years of age and had 1 to 3 positive nodes, removal of $\geq 10$ nodes compared with $<10$ nodes resulted in 10-year survival improvement of $11 \%$.

Table 3 shows survival outcomes according to age and a cutoff of 10 nodes excised in node-negative women and women with 1-3 nodes positive. The comparison of survivals in relation to polychemotherapy and number of nodes removed is not meant to be quantitative and is described only to bring attention to the fact that the survival differences are in a similar range.

Previously reported prospective trials and retrospective studies of the role of axillary node surgery on survival can be divided into those that compared AD to no $\mathrm{AD}$ (all or none) and those that compared variable numbers of nodes excised. As mentioned in the introduction, six randomized trials comparing $\mathrm{AD}$ to no $\mathrm{AD}$ all showed a survival rate that was higher in the group of patients who underwent AD than in the group who did not. Results of three of the trials (including the B-04 trial) did not reach statistical significance, and each of the reports of these trials appropriately concluded that there was no significant difference. However, when the data are considered collectively, there is an approxi- 
mately $5.4 \%$ survival benefit associated with axillary node resection that is statistically significant. ${ }^{9}$

Bland et al. ${ }^{11}$ recently reported the results of a large retrospective study of the National Cancer Data Base information for 547,847 women with breast cancer treated in hospitals in the United States. They recognized that omission of axillary node dissection was increasing over time and that this was particularly true for women with lower incomes, in certain regions of the United States, and at hospitals with lower breast cancer case loads. Of particular importance was the observation of significantly better 10-year survival for patients who underwent an axillary dissection than for those patients who did not. For example, 10-year survival among stage I patients treated with partial mastectomy and $\mathrm{AD}$ was $85 \%$, versus $66 \%$ among comparable women for whom $\mathrm{AD}$ was omitted. These differences are not trivial, and the authors concluded that "lymphadenectomy is therapeutic and diagnostic." They also noted, however, that "sentinel node biopsy may represent an acceptable compromise, but it is still being validated and should be performed only in the context of carefully designed clinical trials."

One of the earliest retrospective studies to evaluate whether survival rates are "influenced by the number of lymph nodes recovered and examined" was reported by Fisher et al. in 1970. ${ }^{12}$ All patients underwent Halsted type radical mastectomy, and 852 patients with pathologically negative nodes were followed for 5 years. Table 4 shows the survival of patients according to the number of nodes identified and the survival at 5 years. These data indicate that in a group of women undergoing the same surgical therapy, a trend toward increasing survival is observed when greater numbers of nodes are removed. This was true for both the node-negative group and the group with one to three nodes positive. The numbers of patients in these two categories were insufficient for the observed differences to be statistically significant.

TABLE 4. Retrospective study by Fisher showing 5-y survival for patients with negative nodes and patients with 1-3 positive nodes stratified by the number of nodes removed ${ }^{12}$

\begin{tabular}{ccc}
\hline & & $\begin{array}{c}\text { 5-y survival for patients } \\
\text { T-y survival for patients } \\
\text { Total no. of nodes }\end{array}$ \\
with all nodes negative & $\begin{array}{c}\text { nodes posive } \\
\text { nodes }\end{array}$ \\
\hline $1-5$ & $74 \%$ & $44 \%$ \\
$6-10$ & $76 \%$ & $58 \%$ \\
$11-15$ & $77 \%$ & $68 \%$ \\
$16-20$ & $79 \%$ & $55 \%$ \\
$21-25$ & $81 \%$ & $68 \%$ \\
$26-30$ & $76 \%$ & $67 \%$ \\
$>30$ & $81 \%$ & $76 \%$ \\
\hline
\end{tabular}

A number of more current retrospective studies have examined the possible effect of the number of excised nodes on survival outcomes. In 2002 van der Wal et al., ${ }^{13}$ concerned about the increasing popularity of $\mathrm{SN}$ procedures and the absence of data on long-term outcomes with this procedure, examined the relationship between the number of nodes removed and survival among 453 consecutive stage I and II breast cancer patients. For patients who were node-negative, the 10-year survival rate was $10 \%$ less when the number of nodes removed was $<14$ than that for those patients who had $>14$ nodes removed. For patients who had pathologically positive lymph nodes, the total number of lymph nodes removed was also a factor significantly associated with survival.

In 2002 Weir et al. ${ }^{14}$ reported on the association between the number of lymph nodes removed during axillary resection and survival for patients with pathologically negative nodes. In a group of 1468 women they found a significantly higher regional recurrence in those who had fewer nodes excised and a trend toward decreased survival. However, the negative impact of excision of fewer nodes appeared to be offset in another group of women, who received systemic adjuvant therapy.

Another analysis was reported by Sosa et al., ${ }^{15}$ who examined the effect of the extent of axillary resection on survival in a group of 464 patients with T1N0 breast cancer. There was a statistically significant difference in survival when groups were analyzed according to a cutoff of $\geq 10$ and $<10$ nodes excised or a cutoff of $\geq 15$ and $<15$ nodes excised. Patients who had $<10$ nodes excised had a $10.5 \%$ lower survival rate than patients who had 10 or more nodes excised. This finding is similar to that of another investigative group, ${ }^{16}$ which noted decreased survival in a group of 452 node-negative women when the number of nodes excised was $<10$.

A somewhat older but larger retrospective review showed differences in survival related to the number of nodes removed when all nodes were pathologically negative. The Danish Breast Cancer Cooperative Group evaluated 13,851 patients registered in its database. ${ }^{17}$ The investigators observed that there was significantly improved disease-free and overall survival in node-negative breast cancer patients who had 10 or more nodes removed.

Two smaller studies yielded different observations. Moorman et al. ${ }^{18}$ compared 255 node-negative breast cancer patients who had $\geq 20$ lymph nodes excised to 656 breast cancer patients who had $<20$ lymph nodes excised. No significant difference in survival was observed. Camp ${ }^{19}$ examined a smaller group of women and observed that 67 women with $\geq 20$ nodes excised had 
worse survival values than 223 women with $<20$ nodes excised.

The observation that the number of pathologically negative nodes in the specimen is related to survival is not limited to breast cancer. A recent retrospective study of patients with rectal cancer showed a significant relation between survival and the number of negative nodes identified in the resected specimen. When the nodes identified in the specimen increased, the observed survival increased. For the first through fourth quartile, 5-year survival rates were $.68, .73, .72$, and $.82 .{ }^{20}$ Also, Chan et al. observed that the extent of lymph node dissection for melanoma, when analyzed by quartiles, is an independent factor in overall survival. Survival was greater among the patients in the higher-quartile noderesection groups. ${ }^{21}$

There are a number of possible explanations for the observation that patients who have more lymph nodes removed have greater survival than those who have fewer nodes excised. One explanation is that patients with more lymph nodes removed are more likely to have a pathologically positive node identified. In this case, patients with fewer nodes are simply being understaged and misclassified. This is unlikely to fully explain the observed differences, because six randomized studies of AD versus observation revealed an overall survival advantage that could not have been masked by misclassification.

However, there are a number of limitations to our data and that from other retrospective studies. Subjective judgments by the surgeons may have impacted the extent of surgery and introduced bias, leading to variable numbers of nodes removed. For example, if the surgeon believed the patient to be at particularly low risk, the extent of regional node resection could be limited. Other types of therapy that could impact the overall resultsfor example, systemic adjuvant therapy-were not controlled in the population that we studied. The number of nodes reported (provided by the SEER database) may not reflect the number of nodes removed. SEER data on resected regional nodes are based on the pathologist's interpretation of the specimen submitted by the surgeon. Although methods vary, usually the pathologist makes a diligent effort to identify and characterize all nodes removed by the surgeon. Thus, at least in reference to existing standard practice, the number of nodes reported by the pathologist generally reflects the number of nodes removed by the surgeon. To some extent, these limitations are mitigated by the very large size of the database.

It is important to realize that pathological negativity does not necessarily mean that a node is entirely free of cancer cells. It means only that to the extent the node was examined, the pathologist did not observe cancer cells. More aggressive techniques to identify cancer cells-for example, serial sections, ${ }^{22}$ immunohistochemistry, ${ }^{23}$ reverse-transcription polymerase chain reaction, ${ }^{24}$ and tissue culture 25 - can result in detection of additional cancer cells in nodes that previously were considered free of cancer. The threshold number of cancer cells necessary to initiate clonogenic growth in a lymph node is simply unknown. It is of course possible that clonogenic tumor cells, albeit sparse, can lead to macrometastatic growth in a node that initially would have been considered negative by pathological examination. Such small numbers of cells may take a considerable period of time to become clinically evident. In support of this notion is the observation that $50 \%$ of locoregional relapses occur after 5 years. ${ }^{26}$

The notion that removal of regional nodes from patients with breast cancer improves survival is strongly supported by both prospective randomized trials and multiple retrospective studies of large groups of women. The magnitude of survival improvement associated with $\mathrm{AD}$ is in the range of benefit observed with systemic adjuvant therapy. That this survival benefit occurs even when all lymph nodes are reported to be negative should be considered a serious issue unless it is disproved by appropriate trials.

\section{REFERENCES}

1. Fisher B, Redmond C, Fisher ER, et al. Ten-year results of a randomized clinical trial comparing radical mastectomy and total mastectomy with or without radiation. N Engl J Med 1985;312: 674-81.

2. Morrow M. Is axillary dissection necessary after positive sentinel node biopsy? Yes! Ann Surg Oncol 2001;8(Suppl):74S-6S.

3. Harris JR, Osteen RT. Patients with early breast cancer benefit from effective axillary treatment. Breast Cancer Res Treat 1985; 5:17-21.

4. Johansen H, Kaae S, Schiodt T. Simple mastectomy with postoperative irradiation versus extended radical mastectomy in breast cancer: a twenty-five-year follow-up of a randomized trial. Acta Oncol 1990;29:709-15.

5. Langlands AO, Prescott RJ, Hamilton T. A clinical trial in the management of operable cancer of the breast. Br J Surg 1980;67: $170-4$.

6. Atkins H, Hayward JL, Klugman DJ, Wayte AB. Treatment of early breast cancer: a report after ten years of a clinical trial. BMJ 1972;2:423-9.

7. Hayward JI. The Guy's Hospital trials on breast conservation. In: Harris JR, Hellman S, Silen W, eds. Conservative Management of Breast Cancer: New Surgical and Radiotherapeutic Techniques. Philadelphia: JB Lippincott, 1983:77-90.

8. Cabanes PA, Salmon RJ, Vilcoq JR, et al. Value of axillary dissection in addition to lumpectomy and radiotherapy in early breast cancer. The Breast Carcinoma Collaborative Group of the Institut Curie. Lancet 1992;339:1245-8.

9. Orr RK. The impact of prophylactic axillary node dissection on breast cancer survival: a Bayesian meta-analysis. Ann Surg Oncol 1999;6:109-16. 
10. Polychemotherapy for early breast cancer: an overview of the randomised trials. Early Breast Cancer Trialists' Collaborative Group. Lancet 1998;352:930-42.

11. Bland KI, Scott-Conner CE, Menck H, Winchester DP. Axillary dissection in breast-conserving surgery for stage I and II breast cancer: a National Cancer Data Base study of patterns of omission and implications for survival. J Am Coll Surg 1999;188:586-95; discussion, 595-6.

12. Fisher B, Slack NH. Number of lymph nodes examined and the prognosis of breast carcinoma. Surg Gynecol Obstet 1970;131:7988.

13. van der Wal B, Butzelaar RM, van der Meij S, Boermeester MA. Axillary lymph node ratio and total number of removed lymph nodes: predictors of survival in stage I and II breast cancer. Eur J Surg Oncol 2002;28:481-9.

14. Weir L, Speers C, D’yachkova Y, Olivotto IA. Prognostic significance of the number of axillary lymph nodes removed in patients with node-negative breast cancer. J Clin Oncol 2002;20:1793-9.

15. Sosa JA, Diener-West M, Gusev Y, et al. Association between extent of axillary lymph node dissection and survival in patients with stage I breast cancer. Ann Surg Oncol 1998;5:140-9.

16. Mathiesen O, Carl J, Bonderup O, Panduro J. Axillary sampling and the risk of erroneous staging of breast cancer: an analysis of 960 consecutive patients. Acta Oncol 1990;29:721-5.

17. Axelsson CK, Mouridsen HT, Zedeler K. Axillary dissection of level I and II lymph nodes is important in breast cancer classification. The Danish Breast Cancer Cooperative Group (DBCG). Eur J Cancer 1992;28A:1415-8.
18. Moorman PG, Hamza A, Marks JR, Olson JA. Prognostic significance of the number of lymph nodes examined in patients with lymph node-negative breast carcinoma. Cancer 2001;91:2258-62.

19. Camp RL, Rimm EB, Rimm DL. A high number of tumor free axillary lymph nodes from patients with lymph node negative breast carcinoma is associated with poor outcome. Cancer 2000; 88:108-13.

20. Tepper JE, O'Connell MJ, Niedzwiecki D, et al. Impact of number of nodes retrieved on outcome in patients with rectal cancer. J Clin Oncol 2001;19:157-63.

21. Chan AD, Essner R, Wanek LA, Morton DL. Judging the therapeutic value of lymph node dissections for melanoma. J Am Coll Surg 2000;191:16-22; discussion, 22-3.

22. Saphir O, Amromin G. Obscure axillary lymph-node metastasis in carcinoma of the breast. Cancer 1948;1:238-241.

23. Weaver DL, Krag DN, Ashikaga T, Harlow SP, O'Connell M. Pathologic analysis of sentinel and nonsentinel lymph nodes in breast carcinoma: a multicenter study. Cancer 2000;88:1099-107.

24. Bostick PJ, Huynh KT, Sarantou T, et al. Detection of metastases in sentinel lymph nodes of breast cancer patients by multiplemarker RT-PCR. Int J Cancer 1998;79:645-51.

25. Heller R, Becker J, Wasselle J, et al. Detection of submicroscopic lymph node metastases in patients with melanoma. Arch Surg 1991;126:1455-9; discussion, 1459-60.

26. Osborne MP, Ormiston N, Harmer CL, McKinna JA, Baker J, Greening WP. Breast conservation in the treatment of early breast cancer: a 20-year follow-up. Cancer 1984;53:349-55. 